CARNETS DE Carnets de géographes

GÉOGRAPHES.

7| 2014

Les espaces de l'entre-deux

\title{
Les espaces intermédiaires et la densification des tissus périurbains
}

Camille Benigni

\section{(2) OpenEdition \\ Journals}

Édition électronique

URL : http://journals.openedition.org/cdg/426

DOI : $10.4000 /$ cdg.426

ISSN : 2107-7266

Éditeur

UMR 245 - CESSMA

Référence électronique

Camille Benigni, «Les espaces intermédiaires et la densification des tissus périurbains », Carnets de géographes [En ligne], 7 | 2014, mis en ligne le 01 décembre 2014, consulté le 19 avril 2019. URL : http://journals.openedition.org/cdg/426 ; DOI : 10.4000/cdg.426

La revue Carnets de géographes est mise à disposition selon les termes de la Licence Creative Commons Attribution - Pas d'Utilisation Commerciale - Pas de Modification 4.0 International. 


\title{
LES ESPACES INTERMEDIAIRES ET LA DENSIFICATION DES TISSUS PERIURBAINS
}

CAMILLE BENIGNI

Architecte-Doctorante en urbanisme et aménagement Centre de recherche sur l'Habitat CRH UMR LAVUE camille.benigni@paris-valdeseine.archi.fr

\begin{abstract}
Résumé
Notre recherche se concentre sur les espaces intermédiaires et leur impact dans un contexte où les politiques urbaines visent une ville " compacte et dense ". En effet, la métamorphose de ces espaces aux limites des sphères du public et du privé est un enjeu fort de ces politiques de densification. Mais comment sont-ils pris en compte dans les procédures et politiques urbaines mises en œuvre sur le territoire ? Nous observerons les formes qui prennent place dans les espaces intermédiaires des tissus pavillonnaires et les utilisations qui y sont associées. Nous faisons l'hypothèse que ces formes architecturales traduisant des utilisations et pratiques sont durables et installées dans une logique inhérente au pavillonnaire. Donner une légitimité à ces espaces "en plus" (Gauthiez, 2003) qui sont investis par les habitants participe à la construction du développement urbain durable (Charmes, 2010), même si nous savons que les contextes législatifs locaux et outils juridiques ont un rôle prégnant sur les possibilités d'investissement de la parcelle. En analysant le devenir des espaces intermédiaires, sur des terrains en lle-de-France, nous escomptons mieux comprendre les processus de densification des tissus périurbains et leur possible planification.
\end{abstract}

\begin{abstract}
Our research focuses on the in-between spaces and their impact in the context of urban policies brought on the density. The densification is one of the goals of "compact and dense" city. What is the role of these spaces between the spheres of public and private in urban policies and procedures implemented in the territory? We hypothesize that these architectural forms resulting practices are sustainable and installed in a logical part of the construction of suburban and residential areas. Give legitimacy to the in-between spaces which are invested by the people contributes to the construction of sustainable urban development for the development of territories, even if we know that the local legislative contexts and tools have a legal role in the investment opportunities of the plot. By analyzing the evolution of these in-between spaces on suburbs in Ile-de-France, we expect to better understand the process of densification of suburban fabric and their possible planning.
\end{abstract}




\section{Introduction}

Dans l'historiographie architecturale, l'apparition de nouveaux édifices est privilégiée, mais l'évolution des bâtiments et des tissus existants n'est pas étudiée dans les différents travaux menés et écrits. De fait, la métamorphose des tissus ne fait pas l'objet de véritables réflexions de recherche.

L'espace intermédiaire est "l'un de ces objets fragiles qu'on aborde rarement en raison d'une complexité de construction, d'un flou ou d'une apparente indétermination, mais qui pourtant nous révèlent beaucoup sur les liens distendus et les affrontements changeants de nos sociétés " (Bonnin, 2005). Ainsi, parce qu'ils disposent d'importants espaces intermédiaires, les tissus constitués d'habitat individuel sont un révélateur des processus de densification et de transformations morphogénétiques à l'œuvre. Ils constituent à ce titre un enjeu majeur pour les politiques de densification. Nous faisons l'hypothèse qu'il y a un décalage entre la place qui leur est faite dans les procédures et politiques et l'ampleur de ce qui se fait en réalité (utilisations et processus).

Depuis le lancement de la réforme de l'Urbanisme et de l'accès au Logement, le vocable de la densification est mis en avant. Comme le souligne Stanley Geneste (2013), "la densification urbaine est désormais régulièrement présentée comme l'un des piliers de l'aménagement durable ". La " loi Duflot " entend dans cette perspective mettre à profit les quartiers pavillonnaires en favorisant leur densification et en encourageant les surélévations. Le dossier de presse publié qui rend compte des principales mesures explique que "les tissus pavillonnaires constituent un gisement de construction de logements favorable à la densification. Pour y parvenir, des limites seront posées à la possibilité, pour les PLU, de fixer une taille minimale de terrain et une densité maximale des constructions, qui sont, trop souvent aujourd'hui, déterminées indépendamment de la morphologie urbaine $"{ }^{1}$. Les politiques urbaines actuelles de l'État estiment que la densification est nécessaire, régulatrice et moteur du développement urbain durable (Charmes, 2010 ; Jenks 2000 ; OCDE, 2012).

La densification ne peut pourtant pas être une simple prescription étatique, car cette politique ne garantit pas une amélioration de la qualité de vie de manière automatique (Da Cunha, 2009). Ses prescriptions restent vagues, elles ne précisent ni des objectifs quantitatifs ni qualitatifs. Il est indispensable de comprendre la manière dont est entendue et définie la densification, notamment telle qu'elle est utilisée et explicitée dans les orientations réglementaires, comme c'est le cas du projet de schéma directeur de l'lle-de-France. De plus, il sera important de préciser les enjeux de la densification à chaque échelle d'intervention. Enfin, il reste toujours une crainte ou des incertitudes sur l'acceptabilité sociale des mesures de densification, surtout aux échelles fines (architecturales ou urbanistiques) du quartier ou de la parcelle. II existe trop souvent un rejet des opérations de densification de la part des habitants (Geneste, 2013).

L'intérêt et la particularité de notre recherche résident dans l'observation et l'analyse des processus de densification "douce $"^{2}$ (Darley, Touati, 2011) qui se sont opérés dans les

\footnotetext{
${ }^{1}$ Lien vers le dossier de presse sur la loi de réforme de l'urbanisme et de l'aménagement pour engager la transition écologique des territoires :

http://www.gouvernement.fr/sites/default/files/fichiers_joints/130429_dp_reforme_urbanisme_duflot.pdf

2 Dans ses travaux sur la politique de densification "douce » des tissus pavillonnaires, sans changer leur morphologie, Anastasia Touati explique que l'habitant constitue le maître d'ouvrage du processus de densification qui en découle. Les outils réglementaires sont à destination des particuliers voire des petites structures de promotion immobilière.
} 
différents tissus pavillonnaires, afin de s'inspirer de ce que les habitants font pour trouver des solutions de densification. En effet, les zones pavillonnaires sont souvent considérées comme la cause de l'étalement urbain, de l'individualisme et soulèvent la question de la préservation des territoires. Les zones pavillonnaires ont été souvent délaissées des politiques urbaines, pourtant la densification y est un processus qui recouvre des pratiques anciennes et variées (Petitet, 2013).

Nous pensons qu'étudier les processus de densification à une échelle, à la fois architecturale et urbaine, et plus particulièrement à l'échelle des espaces intermédiaires de ces quartiers pavillonnaires constitue un prérequis nécessaire à une politique urbaine de densification. En tant que supports de la densification, les espaces intermédiaires permettent de comprendre les appropriations qui naissent de ce processus, mais aussi ses limites. Notre recherche s'intéressant au rôle des espaces intermédiaires pavillonnaires dans les processus de densification, les terrains d'étude retenus témoignent de ce processus, dans ses différentes expressions.

Nous interrogerons donc, dans un premier temps, la définition et le rôle de ces espaces, que nous considérons comme des espaces appartenant à l'espace privé, visibles de l'espace public (Gauthiez, 2003) afin de montrer l'intérêt et d'affirmer la place des espaces intermédiaires pavillonnaires dans le développement des villes. Dans un second temps, pour construire notre typologie des formes d'espaces intermédiaires, il nous a été nécessaire de choisir des terrains d'étude, que nous préciserons. De nos analyses de terrain, nous classerons les formes et utilisations observées afin d'énoncer notre typologie morphologique d'espaces intermédiaires des tissus pavillonnaires. Enfin, ces dispositifs prennent place dans les espaces intermédiaires. Ils participent de la densification des tissus pavillonnaires, nous étudierons les processus de densification dans la dernière partie de notre article.

\section{Les espaces intermédiaires dans les tissus pavillonnaires}

\section{Quelle définition des espaces intermédiaires?}

Au cours du XIX siècle, avec l'écriture des premiers traités d'urbanisme et la mise en place des réglementations urbaines, se forme un corpus de textes, abordant la question des espaces situés entre la rue et le logement et de leur définition (Flamand, 2005). La question de l'articulation entre espace public et privé et celle des relations entre ces sphères se posent rapidement. Dans cette quête architecturale et urbaine de la fin du XIX siècle, teintée d'exigences hygiénistes, plusieurs de ces lieux à la frontière des deux sphères publiques et privées sont l'objet de réflexion et de débats. Nous pensons à la cour, à laquelle de nombreux travaux sont consacrés et pour laquelle des réglementations sont établies ou encore la coursive, considérée comme un lieu de danger (hygiénique et physique).

Pour autant, le sens que revêt l'expression " espace intermédiaire " est fonction du contexte historique, théorique, disciplinaire et idéologique et la variété terminologique est importante: parties communes, espaces collectifs, espaces hors logement, dégagements, extérieurs du logement, prolongements extérieurs, espaces verts, espaces publics de proximité, espaces semi-privés, espaces de transition, articulations, annexes du logement, abords du logement, sas, palier, seuil. La pluralité des expressions et leur ambivalence évoquent des sens différents. Pour notre part, nous retenons le terme d'espace intermédiaire, car avec Amélie Flamand, nous pensons qu'il est neutre et générique (A. Flamand, $2008: 27$ ). Ces espaces ne bénéficient pas d'une reconnaissance officielle, d'un 
point de vue juridique, institutionnel. Ils sont analysés de façon statique et normative et ne sont pas abordés comme des lieux dynamiques comme une articulation entre typologie architecturale et morphologie urbaine, comme le souligne Claire Lévy-Vroélant :

"L'expression espaces intermédiaires relève clairement du vocabulaire des professionnels de l'architecture et de l'urbanisme, faute que les représentations vernaculaires de l'espace urbain et de l'habitat soient en mesure d'offrir des images bien établies et un statut reconnu des divers lieux auxquels cette expression renvoie ". (Lévy-Vroélant, Dussart, Frey, 2003 : 18).

Dans la construction de notre définition et de notre typologie des espaces intermédiaires, une revue littéraire et scientifique a été conduite. Les définitions données de l'espace intermédiaire le caractérisent de façon significative comme une zone d'entre-deux dans une dialectique du rapport public/privé. Elles sont généralement tournées vers le rapport de l'habitant à son espace. Dans le cadre de nos travaux sur la densification des tissus périurbains, nous aborderons l'espace intermédiaire tel que le définit Bernard Gauthiez: " espace aménagé de façon à répondre aux exigences du rapport public-privé. II s'agit généralement d'espaces privés visibles de l'espace public (balcons, couvertures en terrasse, etc.)» (2003: 449). La construction de la typologie d'espaces intermédiaires au travers des formes qui les caractérisent, implique « une pratique privée interagissant avec l'espace public ». Notre recherche s'accordera avec la définition d'Amélie Flamand "les espaces intermédiaires sont des espaces de statut juridique privé mais d'usage commun » (2005: 27).

\section{Avatars}

Dans les années 1990, la notion de "limite » est de plus en plus présente dans la réflexion urbaine au détriment peut-être de celle d'“ espace intermédiaire ». Dès 1995, apparaît la notion de résidentialisation, qui fait débat à propos des grands ensembles et qui « constitue le dernier avatar linguistique en date de cette question aporétique et mythique des espaces intermédiaires, en en attendant alors de nouveaux " (Moley, 2003 : 154).

Mise en place comme une mesure politique de protection, voire policière dans l'habitat collectif, elle pose la question du contrôle, des travaux, du gardiennage et du nettoyage. L'objectif principal affiché par la loi $^{3}$ est de définir les statuts juridiques de ces espaces. Pourtant, malgré leur identification, leur délimitation entre les immeubles et les espaces publics ou rues, l'ambiguïté du statut des espaces intermédiaires demeure. Les découpages et aménagements effectués le sont-ils à des fins d'ouverture ou au contraire de fermeture ? Ont-ils été pensés à partir de la rue ou de l'immeuble?

Cette question des limites, interrogée par le phénomène de résidentialisation, se retrouve dans les périphéries où la démultiplication des limites et des espaces intermédiaires est plus visible et multiforme. Dans les tissus pavillonnaires, qu'ils soient en diffus ou en groupé, souvent moins dense, la verdure s'immisce et la référence à l'idéal pavillonnaire s'exprime : espaces délimités sur l'avant de l'habitation, jardins de derrière ou aux cœurs des résidences. Le phénomène de résidentialisation confère un statut de limite aux espaces intermédiaires. Malgré l'aspect sécuritaire dans les ensembles collectifs qui peut se dégager d'une telle mesure, elle entre dans un processus de remodelage et de transformation des tissus, nous pensons aux espaces intermédiaires. Dans le modèle dominant pavillonnaire,

\footnotetext{
${ }^{3}$ Loi d'orientation et de programmation pour la ville et la rénovation urbaine du $1^{\text {er }}$ août 2003 et Loi de programmation pour la cohésion sociale du 18 janvier 2005.
} 
ces lieux "posent des problèmes ordinaires d'usage, de gestion et d'entretien " (LévyVroélant, Dussart, Frey, 2003 : 18). Une interrogation demeure sur l'existence de dérives à ce phénomène, notamment avec l'essor des lotissements clos ou gated communities en France (Le Goix, 2005). En effet, dans ses travaux sur les gated communities, l'auteur montre que les habitants de ces ensembles pavillonnaires clos seraient à la recherche d'une tranquillité à l'écart des nuisances que l'on trouve dans les grands ensembles (circulation intempestive, regroupement d'adolescents), les accès y sont contrôlés et l'espace public est privatisé (Le Goix, $2005: 1$ ). La place des espaces publics ouverts et des espaces de transition y reste très limitée, à l'exception des zones de circulation (Callen, 2011 : 106), ainsi, notre recherche se concentrera sur d'autres types de transformations, moins radicales, qui s'inscrivent dans des processus visant le développement durable des territoires.

\section{Des espaces qui posent problème}

Les analyses et les travaux de recherche menés par des professionnels, architectes et urbanistes sur les espaces intermédiaires, révèlent que, dans le cadre du logement social collectif, ces espaces bien souvent résiduels y sont définis par défaut (Lévy-Vroélant, Dussart, Frey, 2005 : 86). Peu nombreux, ils sont fréquemment considérés comme des " espaces de renvoi " souvent mal conçus, trop petits et mal situés. La conception, la qualité et la gestion des espaces intermédiaires dans l'habitat collectif, sont souvent victimes d'une politique restrictive de la maîtrise d'ouvrage ou des bailleurs (Lebois, 2011 : 296). Dans les grands ensembles, on y dénombre le développement de pratiques inappropriées et illégales (Lévy-Vroélant, Dussart, Frey, 2005 : 86). Les espaces intermédiaires ne sont pas seulement le support de l'élaboration des relations de l'habitant à son espace mais également le support des relations de voisinage et du rapport entre les habitants, ils ne doivent pas avoir un rôle d'obstacle.

D'une façon générale, dans les tissus pavillonnaires, l'insertion des équipements est difficile et cela pose la question de leur fonctionnement et de leur rentabilité. Cependant lorsqu'on mentionne ces difficultés, caractérisant l'" archaïsme pavillonnaire " (Haumont, 1966), on dénonce non seulement l'organisation urbanistique, mais aussi et surtout l'aliénation des espaces intermédiaires. L'espace du pavillon est caractérisé par son marquage (clôture) qui permet un fort sentiment d'appropriation. Cependant cette appropriation s'effectue sur un espace-temps qui est celui d'une vie. L'habitant a le sentiment de pouvoir avoir sa propre organisation de l'espace. Cet investissement et le développement de pratiques et de dispositifs sur les parcelles pavillonnaires offrent « un autre type de terrains libres : le jardin de devant " (Darin, 2009 : 439). Le jardin de devant dans le tissu périurbain pavillonnaire offre la possibilité d'investir l'espace et donne forme à cet espace intermédiaire entre la rue stricto sensu et l'habitation, il est "le glacis de la maison " (Léger, 2010 : 34). Le jardin constitue un espace à part entière entre le dedans et le dehors, car sans être dans le pavillon, il fait partie du chez-soi et a donc cette particularité d'être un espace de transition vers l'intérieur. Pour les enfants, il constitue un espace extérieur à l'intérieur de la zone de marquage du pavillon, propice à l'intimité de la vie familiale (Tapié, 2005 : 64) et à l'écart de la rue, qui pour les habitants représente toujours un danger.

Aussi, le système pavillonnaire, marqué par la permanence des clôtures, montre un besoin de différencier son espace de vie et d'être tranquille chez soi. II révèle une volonté de contrôler son voisinage et son environnement direct. Les zones pavillonnaires, par leurs jardins, offrent des possibilités d'appropriation qui semblent difficiles dans les grands ensembles. 


\section{Choix des échantillons d'étude}

La popularité française de la maison individuelle et de son jardin est telle que $87 \%$ des Français plébiscitent I'habitat individuel $^{4}$ et qu'aujourd'hui $57 \%$ des Français habitent une maison individuelle isolée (sources INSEE/SOFRES ${ }^{5}$ ). Le type d'habitat individuel reste "neutre en lui-même» (Frey, 1989), ce qui nous permet de nous focaliser sur la transformation de ces tissus et l'étude des espaces. Nos terrains d'analyse rendent compte d'une grande diversité de situations typologiques de tissus périurbains: ensembles pavillonnaires des années 1980-1990, des années 1950-1970 et anciennes zones de villégiature (Raymond, 1966) devenus lotissements ou centre dense. Une particularité méthodologique a été de nous intéresser à des tissus peu étudiés comme le pavillonnaire résiduel des tissus denses, le pavillonnaire à forte valeur patrimoniale ou encore les nouvelles extensions en périphérie (Geneste, 2013). En outre, l'existence explicite d'une politique urbaine relative à nos objets de recherche, à savoir la densification et l'économie de l'utilisation du sol, a guidé nos choix des terrains d'étude.

Pour chaque terrain, différents variables ont été considérées. Tout d'abord la situation urbaine par rapport à Paris (première couronne, moyenne couronne, proximité ou éloignement du centre de Paris, accès transports en commun) ${ }^{6}$. A cet égard, notre échantillon est représentatif d'une diversité de situations géographiques au sein du périmètre francilien. Ensuite, le deuxième critère est la typologie de tissus et la typologie du lotissement (année de construction, situation centrale ou non par rapport au reste de la commune choisie). Le troisième critère concerne la typologie des parcelles et leur évolution morphologique par différents processus de transformation observés. Enfin, un dernier critère entre en compte: la disposition des habitats individuels sur leur parcelle et l'évolutivité des parcelles et de l'habitat qu'elle autorise.

\section{Quelle typologie des formes d'espaces intermédiaires?}

Donner une légitimité aux espaces « en plus " qui sont investis par les habitants participe à notre sens à la construction des solutions durables pour le développement des territoires, même si nous savons que les contextes législatifs locaux et outils juridiques ont un rôle prégnant sur les possibilités d'investissement de la parcelle.

"Les pratiques non logées dans ces univers auront tendance à s'externaliser, à opérer des débordements sur cet espace intermédiaire apparemment moins défini, à l'occuper et à lui ôter ses qualités pourtant indispensables déclenchant en cascade d'autres manques et d'autres conflits " (Bonnin, 2005 : 232).

Les pratiques sont entendues comme "[...] l'ensemble des actions des hommes dans l'espace, consistant simultanément à lui donner des configurations spatiales matérielles et des significations " (Lefebvre, 1970: 203). Cette citation illustre la différence entre les pratiques urbaines et les pratiques urbanistiques, entre, " ce qui relève du vécu et du perçu

\footnotetext{
4 Source Etude «Les Français et leur habitat : Perception de la densité et des formes d'habitat ", menée pour l'Observatoire de la Ville, par la TNS, SOFRES en janvier 2007, http://www.tnssofres.com/sites/default/files/150207_ville.pdf

${ }^{5}$ Ce chiffre est issu du sondage, Les Français et leur habitat : perception de la densité et des formes d'habitat effectué pour l'Observatoire de la Ville par la TNS Sofres en janvier 2007.

${ }^{6}$ Les communes d'étude sont: Colombes, Champigny-sur-Marne, Croissy-sur-Seine, Levallois-Perret, Le Vésinet, Montreuil-sous-Bois, Noisy-le-Grand, Gif-sur-Yvette.
} 
par les habitants et les pratiques d'aménagement des professionnels de l'aménagement » (Semmoud, $2001: 9$ ).

La construction de notre typologie s'est faite d'après l'observation empirique des différents terrains, afin d'être la plus exhaustive possible et, de refléter ainsi la complexité des espaces intermédiaires dans les tissus périurbains pavillonnaires (par la méthode d'observations in situ, d'interprétation des photographies aériennes et relevés de terrain, outil cadastral, Géoportail). Nous avons étudié sur l'ensemble des échantillons d'étude environ 1090 parcelles. En effet, comme le dit Françoise Fromonot, " une maison est une machine à observer » (2009 : 24). Notre méthodologie s'inspire des travaux menés par Jean-Pierre Frey (1991) sur les types architecturaux de logements patronaux (1987) ainsi que de ceux de Nicole Haumont sur les espaces extérieurs du logement, et plus particulièrement les vérandas (Haumont et al., 1988). Ces travaux sont marquants pour la méthodologie de construction et d'élaboration de la typologie étudiée et de sa mise en perspective dans les processus de transformation morphologique des tissus urbains. Certes, ces travaux portent sur des configurations d'espaces étudiés à une autre époque mais l'ambiguïté de leurs statuts et configurations demeurent dans les usages contemporains.

Notre repérage systématique des formes qu'accueillent les espaces intermédiaires dans les tissus pavillonnaires étudiés nous a permis de dégager une typologie de dispositifs spatiaux et d'appropriation à l'échelle de la parcelle, en nous intéressant aussi bien aux " espaces privatifs en extension externe du logement ", qu'à l' « espace interposé entre la résidence et l'espace public » (Moley, 2003). En recourant à la « décomposition de l'organisation spatiale en éléments susceptibles de nous renseigner au plus juste " (Frey, 1987), l'objectif est d'identifier à partir de cette classification les différents processus touchant les espaces intermédiaires.

Nous nous concentrerons sur les espaces situés entre deux pavillons individuels (en limite parcellaire), ainsi qu'à tous les espaces non construits autour du pavillon, qui ont vocation à être investis ou exploités par les habitants ou propriétaires. Nous examinons entre autres le recul de l'édifice par rapport à la rue car il participe de l'« étoffement des espaces intermédiaires ", comme nous le fait remarquer Jean-Pierre Frey dans son étude sur le Creusot (Frey, 1988: 206). Pour chaque forme étudiée, faisant partie de notre typologie d'espaces intermédiaires, nous avons souhaité l'illustrer par un schéma récapitulatif.

\section{Les annexes}

Au sein de notre typologie d'espaces intermédiaires, le premier dispositif observé concerne ce que nous appellerons "les annexes"(Frey, 1987: 123). Parce qu'elles ne sont pas intégrées dans l'édifice, elles y sont définies de la façon suivante, "les véritables annexes sont soit accueillies dans des constructions légères -en appentis accolés en pignon ou sur l'arrière des édifices, ou logées sous les volées d'escaliers extérieurs-, soit architecturées dans des édicules à part, en général en limite de parcelle, éventuellement regroupés sous un même toit, à cheval sur les limites de plusieurs parcelles contigües ». Les annexes sont des objets complexes et ambigus en raison des variations de leurs usages et de leur mode de construction, leur statut reste ambigu, pour le programme qu'elles développent et la forme qu'elles peuvent prendre, cette citation de J.-P. Frey illustre cette complexité :

"Les annexes ont un statut ambigu, moins à cause des variations de programme de leur composition [...] ou du fait qu'elles sont tantôt en appentis, tantôt dans des édicules séparés de l'habitation et font de l'objet d'une combinatoire dans leurs modes de regroupement, que parce qu'elles correspondent à l'adjonction de ce que le 
logement proprement dit n'intègre pas. Elles sont en somme, selon le cas, un plus et un moins dans l'habitat".

Trois catégories d'annexes ont été examinées: les abris de jardin, les garages et les vérandas. Les abris de jardin

Dans les différents tissus observés, nous avons identifié une diversité des configurations mises en place par les habitants: des constructions légères en arrière de la parcelle, des constructions en " dur » ou encore des éléments préfabriqués (chalet ou cabanes de jardin). Les abris de jardin accueillent des activités différentes selon les besoins des habitants. Ils peuvent être des remises pour les outils ou d'extérieur ou bien une pièce pour le bricolage, un atelier.

La configuration de l'abri de jardin la plus fréquemment identifiée est la disposition d'une construction en dur avec une toiture, ayant un style architectural semblable à la construction du pavillon individuel ou alors un abri de jardin préfabriqué. Cet abri de jardin prend place en fond de parcelle et en limite séparative (ensemble des limites parcellaires). L'abri de jardin est "le premier degré de rupture de l'équilibre originel » (Léger, $2010: 34$ ) car il est toujours à la limite de la parcelle et donc au contact des voisins.

\section{Les garages}

Figure 1 Configurations des garages (indépendants, en sous-sol ou en rez-de-chaussée)
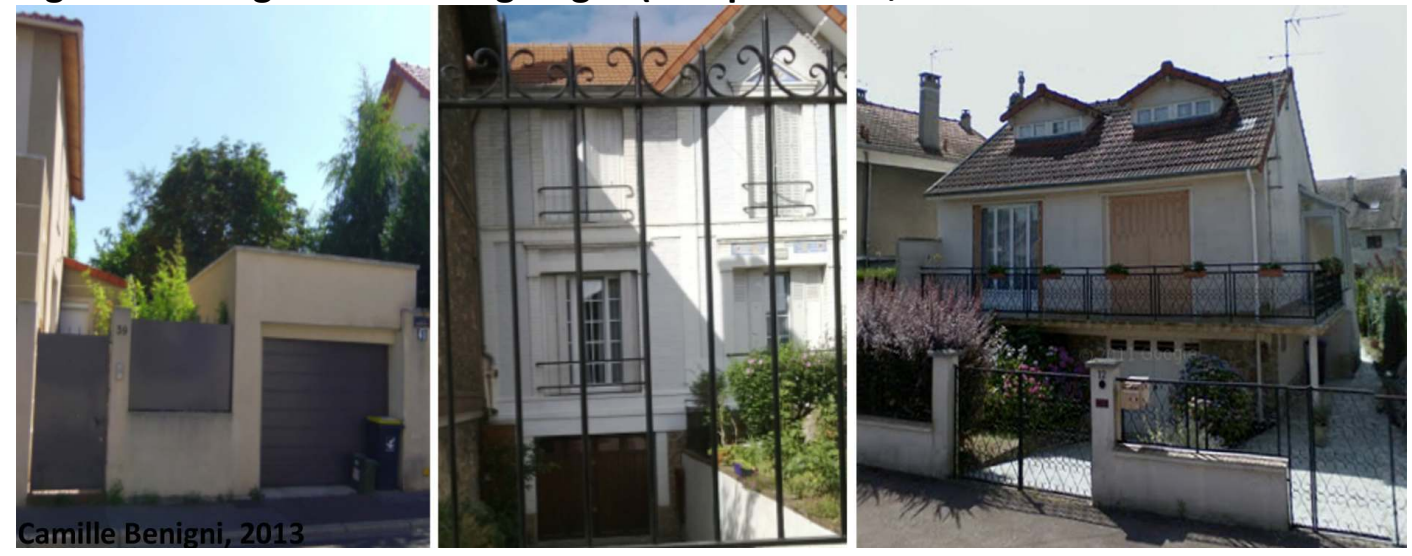

Une autre grande catégorie identifiée est le garage pour voiture. Sa caractéristique est la possible mitoyenneté avec le pavillon. Il constitue ainsi une extension du bâtiment principal, qui se développe soit à l'avant du bâtiment, dans l'espace dédié au recul d'alignement ou au jardin de devant, soit sur une façade pignon entre le pavillon et la limite séparative. Le garage peut également être détourné de sa fonction principale, pour devenir une pièce d'appoint pour le rangement ou l'entreposage. Comme l'illustre cette citation de Françoise Fromonot, " on peut bricoler au garage en débordant sur la chaussée, cuisiner en été sous un feu au pied du talus, faire pousser près des clôtures des fleurs et des légumes (...) " (Fromonot, 2009: 29). Lorsque le garage est une structure indépendante du corps du pavillon, il peut prendre place au fond de la parcelle. Nous avons rencontré quelques garages en "construction légère ", mais le plus grand nombre est construit en dur. Une particularité des pavillons construits dans l'après-guerre est d'intégrer le garage à la construction initiale, soit en sous-sol, soit en rez-de-chaussée. L'entrée du pavillon se situe alors souvent au niveau supérieur auquel on accède par un escalier et un perron. 


\section{Les vérandas}

Une troisième catégorie d'annexes a pu être identifiée: la véranda. Au XIX ${ }^{e}$ siècle, la production industrielle des matériaux prend naissance; la production du verre et les structures métalliques font leur apparition (Haumont, Dussart, 1988). Les vérandas sont d'abord nommées serres, elles s'érigent en espaces de loisirs et de rêves. Elles sont une représentation du développement industriel et de la "nature maîtrisée » (1988: 31). Elles marquent une mutation dans le paysage urbain et périurbain. Les concepteursprofessionnels sont à la fois dans une logique de transparence et de lumière et de démonstration de progrès technologique. Dans l'habitat bourgeois du XIX ${ }^{\mathrm{e}}$ siècle, les serres et espaces vitrés ont une fonction ornementale et d' " agrémentation ». Aujourd'hui, quelques adaptations ont été réalisées et nous observons un engouement pour les espaces vitrés dans l'habitat qui traduit notamment une recherche de performance énergétique de I'habitat, une meilleure isolation de l'ensemble de l'habitation. Une véranda peut apporter une qualité énergétique au bâtiment si la conception architecturale respecte les règles techniques d'orientation, de forme et d'usage. Le succès des vérandas est tel qu'on peut, avec Nicole Haumont et Brigitte Dussart, nous demander si elles ne sont que des gadgets ou une mode éphémère ou bien si elles ne sont pas plutôt un nouvel élément participant à la construction de la typologie d'espaces intermédiaires dans les tissus périurbains.

\section{Figure 2 Dispositifs de véranda adjacente au pavillon individuel à Champigny-sur-Marne}

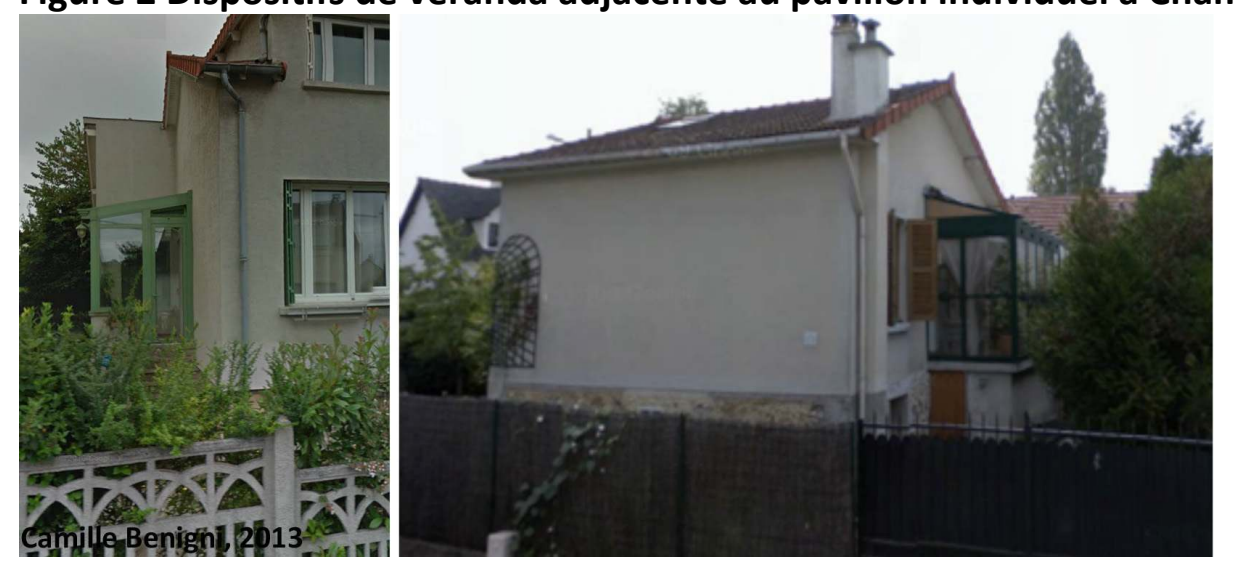

Nous précisons en préambule de notre observation que l'emplacement des vérandas est d'abord fonction des contraintes et des potentialités offertes par l'habitat existant et dépend des dimensions des espaces disponibles entre la rue et l'habitat existant. Lorsqu'elle est placée sur le devant, elle procure une protection aux habitants qui l'ont fait installer ; elle distancie l'habitat et la vie domestique du monde extérieur. Elle l'isole de l'extérieur au sens propre comme au sens imagé. Lorsque la véranda est placée devant la porte d'entrée du pavillon, et donc nécessairement sur le devant de la parcelle et à l'avant de l'habitation, elle devient un dispositif de substitution pour l'accès à l'habitat.

La disposition la plus courante observée est la terrasse transformée en véranda, l'accès à la maison est ainsi protégé du froid et des diverses intempéries. Nous reprendrons ce qui a été dit par Nicole Haumont et Brigitte Dussart, la véranda renforce la "privatisation ", elle permet le contrôle des relations de voisinage des habitants. La véranda semble être une garantie de la préservation de la vie familiale, de son indépendance. Pour les habitants qui décident de l'implanter, la véranda est un véritable espace d'entre-deux entre la sphère publique et l'espace privé. Une autre disposition largement observée est la disposition de la véranda à l'arrière du pavillon ou bien sur un pignon du pavillon. Cet espace devient une 
pièce supplémentaire de la maison, la véranda peut être considérée comme une extension de la maison sur les espaces interstitiels extérieurs qui participent d'une dialectique public/privé. Les habitants la considèrent comme une fermeture du logement par l'appropriation des espaces extérieurs, tout comme la multiplication des dispositifs d'entrée comme les sas, les porches d'entrée, le jardin de devant, entrée. La véranda est une ouverture visuelle sur l'espace environnant ou l'espace de représentation, elle fait partie de la typologie de la maison, elle n'est pas un effet de mode. Considérée par les auteurs de l'étude comme un espace "en plus " ou un espace " de liberté », elle nous apparaît comme faisant partie des formes prises par les espaces intermédiaires. Pour conclure ce développement sur les différentes formes des annexes, nous synthétiserons notre propos par l'illustration d'un schéma récapitulatif.

Figure 3 Schéma récapitulatif des annexes

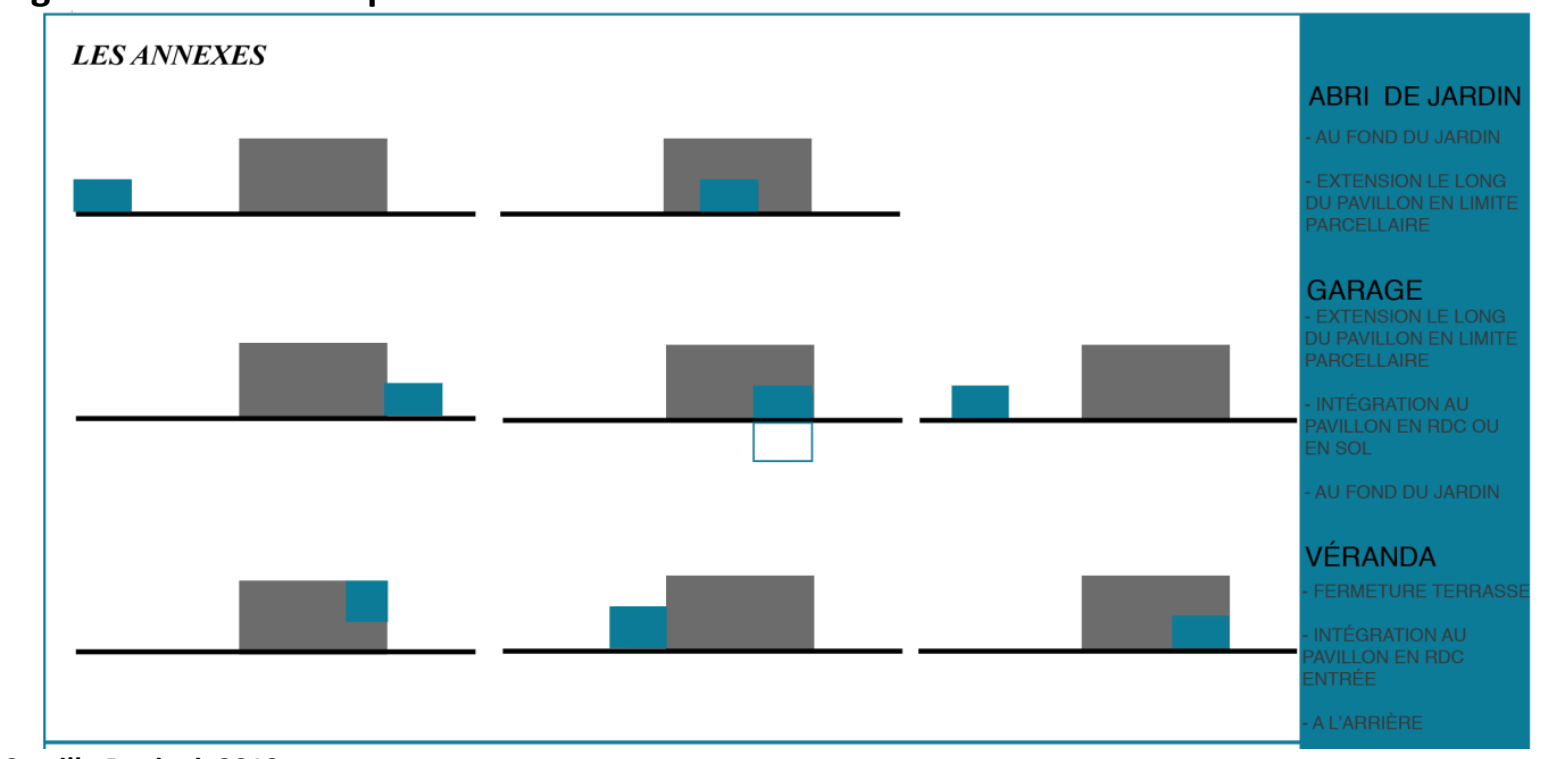

Camille Benigni, 2013

Dans notre recherche typologique des dispositifs spatiaux formant les espaces intermédiaires, nous nous sommes intéressés, outre les annexes aux avancées de toiture que sont les appentis, aux perrons, ainsi qu'aux clôtures et aux toitures proprement dites.

Les appentis

Un appentis est un toit à un seul versant dont le faîte de la toiture s'appuie sur ou contre un mur, en l'occurrence, le mur du pavillon. Il correspond à un prolongement de toiture ou à la création d'une nouvelle toiture (figure 4). Pour les appentis également, nous avons pu repérer une variation des fonctions selon les besoins des habitants. La fonction principale d'un appentis est l'abri pour la voiture, mais il peut aussi servir à abriter le bois de chauffe ou encore le mobilier de jardin, pour devenir un espace de repas en été. L'appentis se révèle être une prolongation spatiale de l'habitation mais, tout comme la véranda, il n'est pas doté d'une fonction définie, il peut accueillir des usages différents de ceux prenant place au sein de l'habitation ou dans le reste des espaces extérieurs.

\section{Les perrons}

Considérés comme éléments décoratifs, les perrons peuvent apparaître comme un élément secondaire. Cependant, comme nous l'explique P. Bonnin, pratiquer l'espace intermédiaire, « c'est quitter un groupe social, un rôle, un statut, une pratique, pour opérer en soi et sur soi 
une transformation afin de se préparer au nouvel espace à venir, aux nouveau groupe, rôle, statut, pratique ou moment de la pratique que l'on va adopter » (2005: 231). Le perron, parce qu'il participe de ce parcours de franchissement et a les caractéristiques d'une limite et d'un seuil (2005: 232), entre dans notre typologie d'espaces intermédiaires. Le perron n'est pas l'élément principal dans la construction de notre typologie mais nous tenterons de comprendre dans quelle mesure, il participe de ce processus de densification en analysant leurs possibles transformations. De nos observations, le perron peut être transformé en véranda ou en sas d'entrée.

Figure 4 Schéma récapitulatif des avancées de toiture

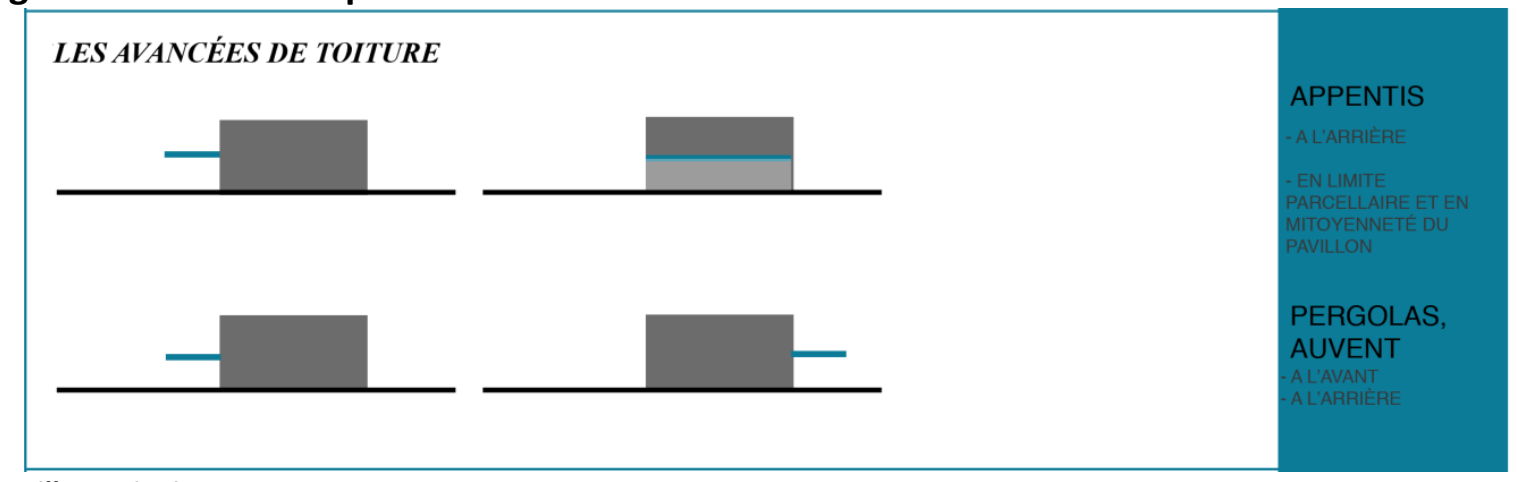

Camille Benigni, 2013

\section{La clôture}

Les clôtures symbolisent le marquage séparatif entre public et privé, car en effet, " [...] la frontière figurée par la clôture démultiplie la possibilité d'autant de contacts que de risques, car les contacts sont à la fois le sel de la sociabilité pavillonnaire (les relations de voisinage pouvant constituer un potentiel équivalent à celui des liens familiaux) et son poivre quand les échanges ne respectent plus le code de bon voisinage " (J.-M. Léger, 2010 : 34). Elles créent des limites dans le paysage.

Nous abordons les espaces intermédiaires entre l'espace public et le logement à proprement dit en nous préoccupant des vis-à-vis et de la mitoyenneté. Ainsi, nous répartirons les catégories de clôtures par configuration : fermée ou ouverte. La clôture est matérialisée par " une palissade, un mur, une séparation grillagée ". Les configurations ouvertes observées, caractérisées par une clôture ouverte sur l'espace public et l'espace privé de la parcelle et du logement, sont les suivantes : muret en pierre naturelle ou pierre meulière surmonté d'une grille, palissade dans les opérations contemporaines, muret béton surmonté d'une grille.

Figure 5 Dispositifs de clôtures fermés et occultant à Champigny-sur-Marne ou clôtures végétales à Gif-sur-Yvette
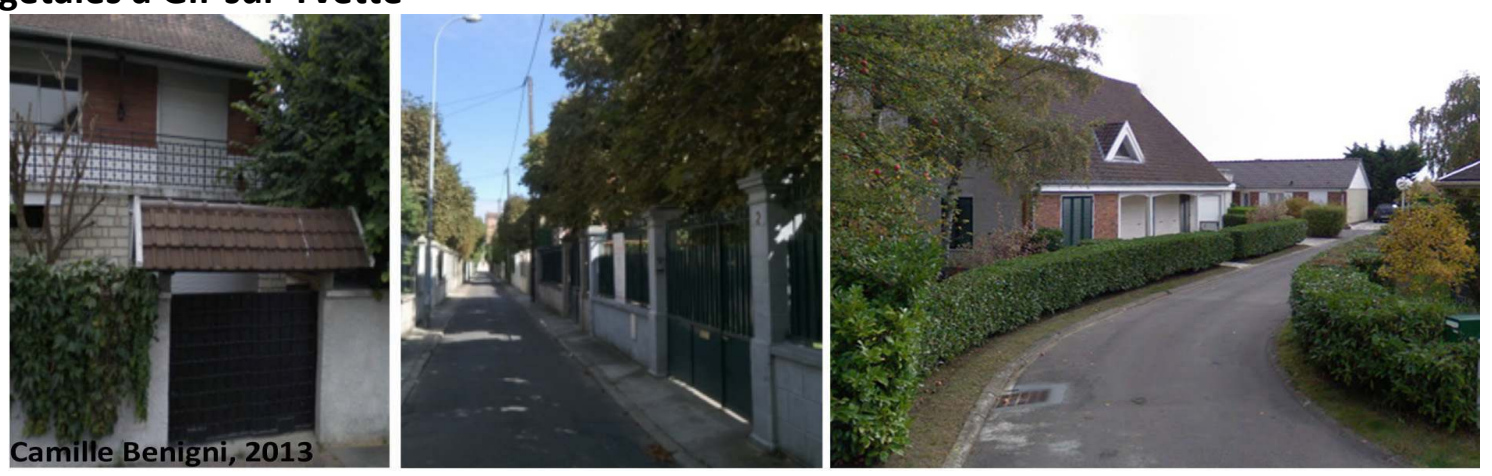
La configuration fermée est identifiable plus facilement par une clôture plus marquée, ne laissant aucune visibilité depuis l'espace public vers l'intérieur de la parcelle et vice versa. Les clôtures fermées peuvent prendre la forme d'un mur plein en pierre ou béton, d'un muret surmonté d'une grille doublée de tôles métalliques, de végétation occultant ou de brise-vue naturel en bambou. Dans les opérations de lotissements pavillonnaires, nous avons rencontré des configurations de clôtures uniquement végétales, qui sont un masque visuel mais ne marquent pas la fermeture de l'espace lorsqu'elles sont basses. La clôture végétale est désignée comme une haie vive, que $P$. Frileux aborde comme "l'élément essentiel de la structure pavillonnaire » (2010:639), car les haies constituent un " maillage vert dont le fonctionnement biologique révèle les pratiques des habitants " (2006). Ainsi, selon les matériaux utilisés, la clôture participe du prolongement d'un espace vers l'autre ou bien de sa fermeture. L'enjeu de la configuration des clôtures en est un au même titre que celui de la porte qui est « le lieu d’un mouvement entre intérieur et extérieur » (Paris, 2005 : 212). La figure 6 ci-dessous schématise les différentes configurations de clôtures étudiées.

\section{Figure 6. Schéma récapitulatif des clôtures}



Camille Benigni, 2013

\section{La toiture du pavillon}

Dans le projet de loi pour l'accès au logement et un urbanisme rénové présentée par Cécile Duflot en mai 2013, la construction sur les toitures des immeubles et la surélévation des pavillons individuels sont à l'ordre du jour afin de participer à une densification douce des tissus urbains et périurbains. Ainsi, nous avons souhaité prendre en considération la toiture comme une forme d'espace intermédiaire. Le grenier est défini comme « un espace résiduel des combles" (Frey, 1987). Son usage premier est caractéristique de l'habitat rural. Un grenier a pour utilisation, le stockage des denrées alimentaires et la relégation d'objets de l'espace domestique. En référence à Gaston Bachelard, dans La poétique de l'espace, J.P. Frey explique que le grenier du pavillon (les combles et sous-pentes du toit) est un « lieu chargé d'une poétique de l'habiter » (Frey, 1987 : 186).

Les combles, entendus comme la superstructure d'un bâtiment comprenant la charpente et la couverture, correspondent au volume compris entre le plancher haut du dernier niveau et le toit du bâtiment. Ils peuvent faire l'objet dans un pavillon individuel d'un aménagement nécessitant leur surélévation afin d'en faire une pièce supplémentaire au dernier niveau. Nous avons travaillé sur le repérage empirique des combles habitables d'après la définition donnée par J.-P. Frey (1987: 115). Les combles habitables ou le grenier peuvent être "susceptible d'accueillir une ou plusieurs pièces supplémentaires ou d'organiser le logement sur au moins deux niveaux est parfois délicat à opérer ». Leur repérage peut être effectué par "la présence d'un escalier d'accès à partir du logement, d'une fenêtre en 
pignon ou d'une lucarne, ou bien d'un volume suffisamment haut sous charpente et accessible d'une façon ou d'une autre (trappe, échelle de meunier), qui permet d'attester l'existence de combles conçus pour un éventuel usage d'habitation ». Ce schéma ci-dessous (figure 7) représente la volumétrie de la toiture par rapport au reste du pavillon.

Figure 7 Coupe Avant / Après Surélévation des combles et extension à l'arrière pour remplacer la véranda.
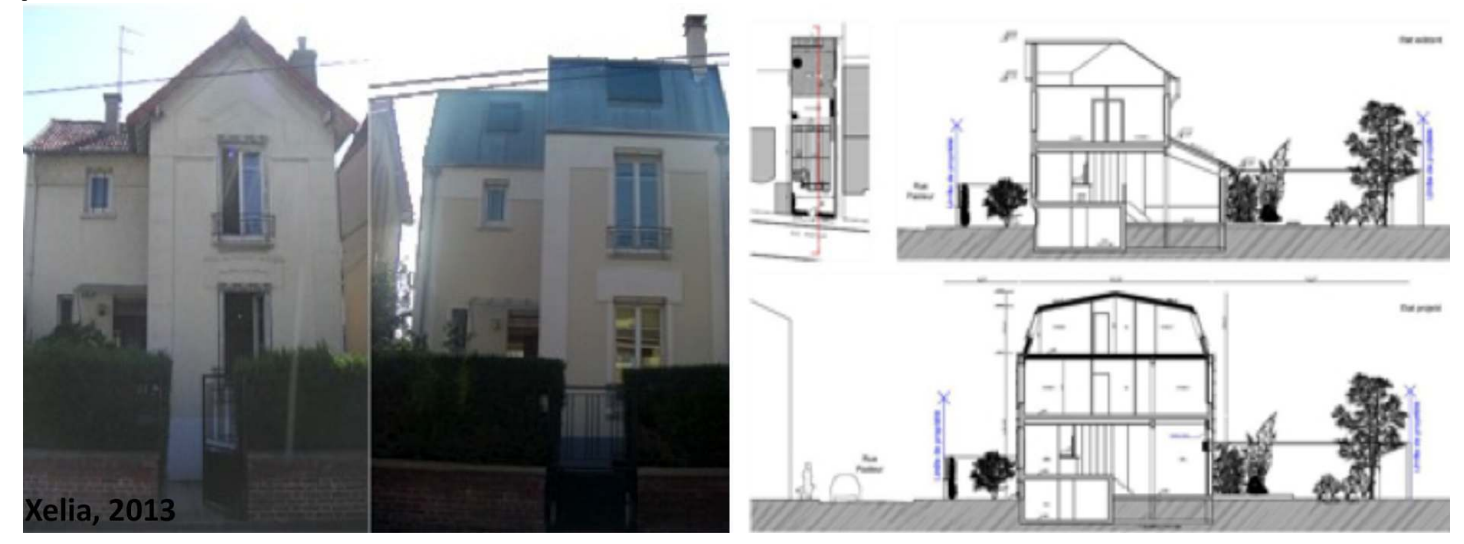

Cette typologie d'espaces intermédiaires met également en avant ce processus de densification, par l'extension horizontale ou verticale du bâtiment.

\section{La densification}

L'observation de l'investissement des espaces intermédiaires et des appropriations qu'ils montrent sur les parcelles pavillonnaires permet d'identifier que le processus de densification est un processus morphologique spontané. II est un "processus d'accroissement de l'occupation d'un site par ajout de bâtiments " (Gauthiez, 2003) qui peut être désigné par le terme "bourrage " (Loyer, 1983) à l'échelle de la parcelle. Il s'oppose à l'urbanisation de nouveaux terrains. Par l'étude des transformations morphologiques des tissus urbains parisiens, au $\mathrm{XIX}^{\mathrm{e}}$ siècle, François Loyer met en évidence la spontanéité des mécanismes de densification à l'échelle de la parcelle, la figure 8 rend compte de ce processus.

Figure 8 Schémas de François Loyer représentant le processus de densification à la parcelle.
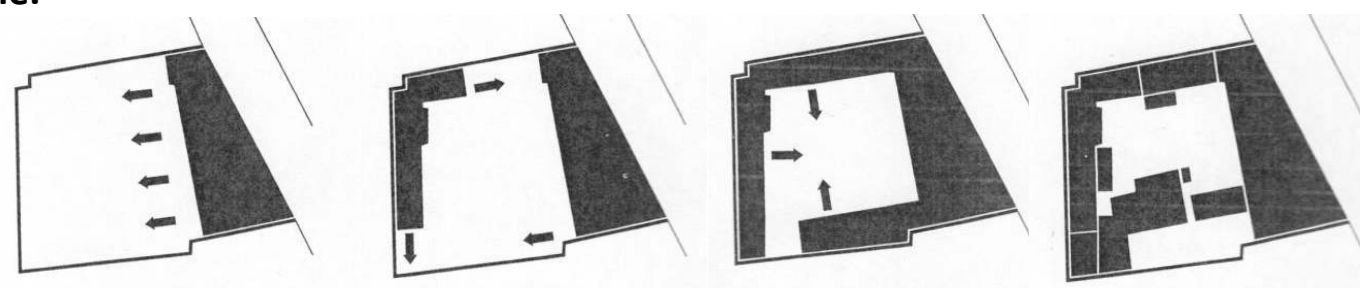

Loyer, $1987: 52$

Depuis une dizaine d'années, nous assistons à des changements de cap en ce qui concerne la politique de densification. Des lois tentent de statuer pour empêcher la densification (comme la loi "Urbanisme et Habitat» qui permettait aux communes d'imposer une superficie minimum aux parcelles) ou pour la favoriser comme la loi SRU en 2000 ou encore la loi Grenelle I et II) à l'échelle locale ou territoriale. En 2000, la Loi SRU est instaurée pour empêcher l'étalement urbain. Pourtant, ces prérogatives législatives n'ont qu'une portée 
locale limitée. L'étude de la morphogenèse des tissus périurbains ouvre en revanche de nouvelles perspectives de transformation et d'aménagement durable des périphéries. C'est pour cela qu'à notre sens les politiques mises en œuvre pour la durabilité des territoires doivent faire émerger cette réflexion. Par ailleurs, la loi Duflot prévoit des études de densification, mais elles ne doivent pas se contenter uniquement de recenser les parcelles disponibles en vue de densifier pour densifier, mais apporter un éclairage sur les processus de transformation morphologique des tissus à l'œuvre, dont les habitants et leur habitat sont au cœur. Là se trouve peut-être la clé stratégique d'une politique de densification durable et réussie.

\section{Observations de la densification par les espaces intermédiaires}

La diminution des tailles des parcelles, pour une configuration d'un pavillon isolé au centre de sa parcelle, n'étant pas imaginable pour limiter l'étalement urbain dans les communes périurbaines, il est nécessaire d'envisager d'autres solutions. Densifier n'est pas s'opposer à la maison individuelle. L'observation des processus de densification observés semble confirmer les propos de J.-M. Léger lorsqu'il écrit que dans les tissus périurbains, l'équidistance de la maison individuelle à ses limites parcellaires représente le degré des relations avec ses voisins pour qu'elles soient pacifiques et qu' « il n'y a pas donc a priori de bonne distance spatiale qui équilibre une bonne distance sociale, l'intrusion étant relative à la distance dont chacun a l'expérience " (Léger, $2010: 34$ ).

Le premier processus de densification relevé est l'extension du bâti qui peut prendre différentes formes, illustrés par la typologie précédemment énoncée. Dans le cas des habitants ou propriétaires les plus modestes, la densification survient après l'accumulation qui a permis d'acquérir ou d'édifier le minimum. Il s'agit donc d'un long processus conduisant au confort (Panerai, 2008). Au cours de cette période, un bâti modeste ou un logement minimal peut devenir un "chez-soi » dans lequel se manifestent les envies, les désirs des occupants, par l'ajout d'une véranda, d'un garage, d'un abri de jardin ou d'un appentis. Cependant cette densification est soumise aux réglementations et aux règles de prospect. Ces règles figent le pavillon au milieu de sa parcelle, les travaux de l'ANR BIMBY ("Construire dans mon jardin : La démarche BIMBY «Build in My Back Yard ») ont suggéré un assouplissement du règlement du PLU (Plan Local d'Urbanisme). En effet, un assouplissement des règles permettrait la construction ou l'adossement en mitoyenneté. Cette construction par addition ou ajouts successifs est un des processus de la densification observée. Elle peut préfigurer « ce que serait la construction d'une nouvelle maison pour les colotis " (Léger, $2010: 34)$.

Un deuxième processus de densification est le phénomène de dédoublement du bâti sur une même parcelle, qui est une autre logique de la densification. Ce processus est ce que nous appellerons le processus d'ajout (construction d'un second pavillon sur la parcelle afin de louer ou vendre), dans le cas de ce processus devient une copropriété. Son ampleur est limitée dans les terrains observés. Appelé "division parcellaire ", notamment dans le cadre de l'ANR BIMBY, il constitue une solution pour densifier les tissus périurbains dans le respect de leurs spécificités morphologiques. Car une densification massive des zones pavillonnaires par division parcellaire paraît difficilement réalisable, voire contre-productif (Petitet, 2013), même lorsque les superficies des parcelles la rendent possible.

Nous aborderons un dernier processus de densification observé, notamment dans des terrains comme Levallois-Perret ou Colombes: les substitutions. Cela consiste en une substitution d'un pavillon par un immeuble de plusieurs logements dont la volumétrie 
générale doit s'intégrer dans le tissu existant. Ces substitutions peuvent amener à réfléchir à des dispositifs d'articulation avec les pavillons existants et à un travail sur les espaces intermédiaires, les accès, les entrées, les espaces extérieurs et mitoyens; sans cela, l'intégration risque d'être compliquée. De telles substitutions sont souvent disséminées dans le tissu en fonction des opportunités et mais elles peuvent être également rassemblées autour d'un axe circulatoire afin de donner une densité plus forte et même d'accueillir des équipements et services qui viseront à améliorer la qualité de vie des habitants, tout en créant de nouveaux logements. La densification au « coup par coup » (Geneste, 2013) mérite de s'inscrire dans une politique visant à préserver les territoires. Ainsi, ce type de densification encadré ne remettrait pas en cause tous les tissus pavillonnaires environnants. II viendrait créer un jeu de complémentarité dynamique avec la ville et serait susceptible de permettre la valorisation des tissus de centres-bourgs en leur réattribuant de la centralité. Ces processus de densification par substitution peuvent également se révéler être des stratégies de promotion immobilière et d'accroissement des prix immobilier et/ou foncier qu'il conviendrait d'étudier davantage. En effet, celles-ci n'engageant que de petites opérations d'aménagement, elles peuvent s'avérer coûteuses pour le contribuable, car non coordonnées (Vilmin, 2008: 82), aussi elles doivent pouvoir être intégrées dans une politique cohérente visant la densification.

\section{Conclusion}

Nous nous sommes intéressés à la typologie d'espaces intermédiaires dans les tissus pavillonnaires en considérant à la fois leur actualité et leurs formes. Les espaces intermédiaires deviennent un enjeu pour la transformation des tissus urbains et périurbains. Il existe sans doute le reformatage systématique des tissus urbains par ces espaces intermédiaires, ils ont un rôle dans ces transformations spontanées ou planifiées. Les espaces "entre-deux" que nous avons choisi d'appeler "espaces intermédiaires" représentent sans doute le lieu stratégique où se joue le processus de fond de fabrication de l'espace urbain. L'observation des tissus pavillonnaires et la construction de notre typologie de formes d'espaces intermédiaires montrent que les tissus se transforment par ces espaces, qui jouent le rôle de soufflets et de joints (Lévy-Vroélant, Dussart, Frey, 2003 : 19). Cette typologie nous permet de donner un cadre explicatif et descriptif des espaces intermédiaires. Les formes que prennent les espaces intermédiaires participent aux processus de densification, comme celui de l'extension du bâti.

La limitation de l'expansion des tissus peu denses et de l'étalement urbain semble inéluctable. La densification des zones pavillonnaires apparaît comme une solution, même si d'un point de vue économique, "la densification présente le risque d'une perte de valeur du bien par diminution de la rente de situation (vue et silence) " (Léger, 2010: 34). L'observation in situ des espaces intermédiaires nous a permis d'identifier des processus de densification des tissus. Les solutions vernaculaires existantes telles que relevées dans notre typologie peuvent inspirer la politique de densification. Les espaces intermédiaires jouent un rôle effectif dans les ajustements à la parcelle. Par notre recherche sur ces espaces $d^{\prime}$ " entre-deux », nous tenterons de préconiser des solutions durables en vue d'évoluer vers une urbanisation soucieuse de l'environnement.

\section{Bibliographie}

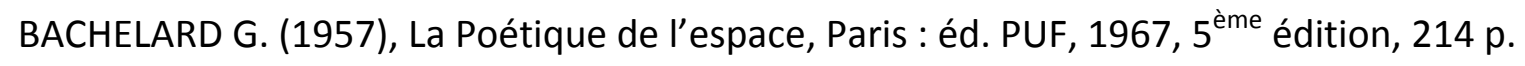


BONNIN P. (2005), " L'Immeuble parisien et sa loge: seuils et rituels des espaces d'articulation " in HAUMONT B. MOREL A. (2005) La Société des voisins, Partager un habitat collectif, Paris : éd. de la Maison des Sciences de l'Homme, $334 \mathrm{p}$.

CALLEN D. (2011), "La fabrique péri-urbaine ", système d'acteurs et production des ensembles pavillonnaires dans la grande couronne francilienne, thèse soutenue le 8 novembre 2011, sous la direction de Denise Pumain, Université Paris I Panthéon Sorbonne, $402 \mathrm{p}$.

CHARMES E. (2010), La densification en débat in Études Foncières, n¹45, mai-juin 2010, pp. 20-23

DARIN M. (2009), La Comédie urbaine, Paris, Éditions Infolio, coll. Archigraphy, Librairie de I'architecture et de la ville, $559 p$

FLAMAND A. (2005), Les Espaces intermédiaires, un état des lieux raisonné, Actes de la journée du GIS socio-économie de l'habitat, édition numérique, décembre 2005, http://resohab.univ-paris1.fr/jclh05/

FLAMAND A. (2008), L'Invention des espaces intermédiaires dans I'habitat, thèse sous la direction de Jean-Pierre Frey, Paris: Institut d'Urbanisme de Paris-CRESSAC-CRH, UMR LOUEST, Paris XII, 2008, $411 \mathrm{p}$.

FREY J.-P., CROIZE J.-C., PINON P. (1991), Recherches sur la typologie et les types architecturaux, Paris : I'Harmattan, $368 \mathrm{p}$.

FREY J.-P. (1987), Société et urbanistique patronale, tome 2 : La généalogie des types de logements patronaux 1836-1939, Paris : Plan MULT/MAIL, $562 \mathrm{p}$.

FRILEUX P. (2010), "A l'abri de la haie dans le bocage pavillonnaire », Ethnologie française, vol. 40, avril 2010, pp. 639-648

FRILEUX P. (2006), "Bocages pavillonnaires périurbains : quelle diversité bio-culturelle ? ", Journées francophones des sciences de la Conservation de la Biodiversité, Le réveil du Dodo II, Paris, 7-9 mars 2006.

FROMONOT F. (2009) « Une petite maison dans le grand Paris », Criticat, n4, pp. 24-37

GAUTHIEZ B. (2003), Espace urbain, vocabulaire et morphologie, Paris, Éditions du Patrimoine, $496 \mathrm{p}$.

GENESTE S. (2013), "Rendre acceptable la densité en tissu pavillonnaire ", in Métropolitiques, 25 mars 2013, URL : http://www.metropolitiques.eu/Rendre-acceptable-la-densite-en.html HAUMONT N., DUSSART B., RETBI M., DIDIER N., TROCHE J.-P., POUGET A. (1988), Concevoir et habiter l'espace de la véranda, Paris, Éditions Plan Construction et Architecture, $144 \mathrm{p}$.

HAUMONT N., RAYMOND H., RAYMOND M.-G., HAUMONT A. (1966), L'Habitat pavillonnaire, Paris, Institut de sociologie urbaine, $148 \mathrm{p}$.

LEBOIS V. (2011), “ L'Entre-deux de l'immeuble parisien » in BERGER M. ROUGÉ L. (2011), Etre logé, se loger, Habiter : regards de jeunes chercheurs, éditions I'Harmattan, coll. Habitat et Sociétés, $322 \mathrm{p}$.

LÉGER J.-M. (2010), "Densification des lotissements : les pavillonnaires font de la résistance ", Études foncières, mai 2010, pp. 33-35.

LE GOIX R. (2005), "La Dimension territoriale des gated communities aux Etats-Unis : la clôture par contrat ", Cercles, n¹3, pp. 97-121

LEFEBVRE H. (1970), La Révolution urbaine, Paris : éditions Gallimard, coll. Idées, 1970, $248 \mathrm{p}$.

LEVY-VROELANT C., DUSSART B., FREY J.-P. (2005), «Entre paradoxe transparence et impossible entre-soi. Les espaces collectifs de la cité Beaumarchais (Montreuil)», in 
HAUMONT B. MOREL A. (2005) La Société des voisins, Partager un habitat collectif, Paris : éd. de la Maison des Sciences de l'Homme, $334 \mathrm{p}$.

LEVY-VROELANT C., DUSSART B., FREY J.-P. (2003), Les Enjeux de la résidentialisation des cités HLM : Analyse des usages des espaces intermédiaires dans une cité de Seine-SaintDenis, rapport final du programme de recherche du ministère de la Culture et de la Communication: Entre privé et public. Les rapports de cohabitation et les usages des espaces communs dans les ensembles résidentiels, $215 \mathrm{p}$.

LOYER F. (1987), Paris XIX siècle : l'immeuble et la rue, Paris : éditions Hazan, 478 p.

MOLEY C. (2006), Les Abords du chez-soi en quête d'espaces intermédiaires, Paris, Éditions La Villette, $256 \mathrm{p}$.

MOLEY C. (2003), Entre ville et logement : en quête d'espaces intermédiaires, rapport pour le ministère de la culture, DAPA, Mission du patrimoine ethnologique, Paris, Éditions La Villette, $180 \mathrm{p}$.

OBSERVATOIRE DE LA VILLE, TNS SOFRES, "Les Français et leur habitat: Perception de la densité et des formes d'habitat ", étude menée pour l'Observatoire de la Ville, par la TNS, SOFRES en janvier 2007, http://www.tns-sofres.com/sites/default/files/150207_ville.pdf

PARIS H. (2005), "L'Inconnu familier, les interactions dans les parties communes d'un immeuble lyonnais ", pp. 209-230 in HAUMONT B. MOREL A. (2005, La société des voisins, Partager un habitat collectif, Paris : éd. de la Maison des Sciences de l'Homme, $334 \mathrm{p}$.

PETITET S. (2013), "Densifier l'habitat pavillonnaire: des démarches individuelles aux projets collectifs ", in Métropolitiques, 20 mars 2013, URL : http://www.metropolitiques.eu/Densifier-I-habitat-pavillonnaire.html

RAYMOND M.-G. (1966), La Politique pavillonnaire, Paris : éd. Institut de Sociologie Urbaine, Centre de Recherche d'Urbanisme, Paris, $360 \mathrm{p}$.

SEMMOUD N. (2001), Les Stratégies d'appropriation de l'espace à Alger, Paris, Éditions I'Harmattan, $271 \mathrm{p}$.

TAPIE G. (2005) "J'habite une maison... » in TAPIE (GUY) (dir.), Maison individuelle, Architecture, Urbanité, Paris : éd. de l'Aube, $251 \mathrm{p}$.

TOUATI A., DARLEY A. (2011) " Processus et politiques de densification de l'habitat en lle-deFrance " in Note rapide de l'Institut d'Aménagement et d'Urbanisme Ile-de-France, $\mathrm{n}^{\circ} 567$, sept. 2011, 4 p., URL : http//www.iauidf.fr/fileadmin/Etudes/etude_866/NR_567_web.pdf

TOUATI A., DARLEY A. (2013) La Densification pavillonnaire à la loupe, dynamiques régionales, stratégies locales et formes urbaines, étude de l'Institut d'Aménagement et d'Urbanisme lle-de-France et du laboratoire LATTS, mai 2013, 77 p., URL :

http://www.iauidf.fr/fileadmin/Etudes/etude_1002/La_densification_pavillonnaire_a_la_loupe.pdf VILMIN T. (2008), L'aménagement urbain en France: Un approche systémique pour construire des stratégies d'aménagement durable,s Paris : éd. Certu, 216 p. 